\title{
Treatment of Trauma through Integrational Dissociation and Distortion
}

\author{
Anil Thomas \\ NLP Trainer \& Gestalt Master \\ Mumbai, India
}

\author{
Kumud Poptani \\ Graduate from Mira's College \\ Raipur, India
}

\begin{abstract}
Trauma has been one of the oldest and most difficult sufferings of humans. Even though the source of trauma has passed, there are long-lasting effects that generations have to deal with. In this research, we explore and validate a treatment method for the trauma that involves the usage of dissociation and distortion in the form of the much-studied V/AKD technique. We discuss the impact of trauma, organisation of traumatic memories, and the V/AKD technique based on the theories of Richard Bandler, John Grinder, and Steve Andreas. 216 participants were tested on the Impacts of Events Scale (R) and further, 50 participants with a mean score of more than 8 , were selected to test the intervention technique which entailed a free-form interview. The results produced have potential usage for additional experimental studies of such intervention procedures and define effective as well as swift treatment methods for pertinent suffering like that of trauma.
\end{abstract}

Keywords: Trauma, Memories, Treatment, V/AKD, Dissociation, Distortion

\section{Introduction}

The following paper identifies the functioning and need for treatment of trauma in people with acute negative life experiences. This study further explores the treatment methods which can be shown effective in this process by understanding the impact and efficiency of these techniques. These techniques include dissociation, distortion and a combination usage of these methods in techniques like the V/AKD (Visual-Auditory Dissociation).

This Research Paper is on 'Treatment of Trauma' and has been authored by Anil Thomas.

This paper has been co-authored by Kumud Poptani, an Intern part of the Global Internship Research Program (GIRP) who graduated from St. Mira's College for Girls, Pune, India.

Kainaz Bharucha served as a Statistical Research Adviser who is an Intern part of the Global Internship Research Program (GIRP).This paper has been critically reviewed and proofread by Shaifila Ladhani.

We thank our Trupti Machwe (Our Editor in Chief) and Priya Pawar (Deputy Editor in Chief) for their dedicated time and contribution towards IJNGP.

Correspondence concerning this article should be addressed to

E-mail: office@ijngp

\section{Trauma}

Trauma has been mostly termed and popularised as an experience in a person's life; it is rather the consequence of an event or experience. It perpetuates after a negative experience in a person's life which leaves them emotionally or physically wounded. We need to understand that trauma stems from and is an after effect of intentional mistreatment of living beings i.e. abuse (mental or physical). While abuse, for example, is the actual event of that distress, trauma is the emotional response to that abuse which gets triggered by the same or similar sets of stimuli repeatedly, making the abused relive the abuse. Trauma results from an event, series of events, or set of circumstances that is experienced by an individual as physically or emotionally harmful or threatening and that has lasting adverse effects on the individual's functioning and physical, social, emotional, or spiritual well-being (Substance Abuse and Mental Health Services Administration [SAMHSA], Trauma and Justice Strategic Initiative, 2012, p. 2).

Supported by Devashish Polymers

Research and Learning Grants' Partner 
Trauma carries the tendency to leave someone with loss of hope, limited expectations about the future and of leading a 'normal' life ahead. According to the PTSD viewpoint, many people develop a long-term mental disorder after experiencing or witnessing horrific events, which is called, "Post Traumatic Stress Disorder" by the DSM-III (American Psychiatric Association, 1980).

The different aspects like the intensity, aspects of each trauma incident, the recurrence interact differently with each person who goes through such an event also depending upon their personality traits and different aspects of their life and self. It is imperative to understand the intricacies that are involved in each perspective of a trauma and how the systems that people are a part of, affect the way they perceive trauma. Once this is understood, the professionals can provide a better trauma-informed treatment to the client (Wallace, 2020). In the treatment method used in the following study, while the details of the event aren't necessary, how the client reacts to each step with respect to the trauma event is of importance.

\section{Memory Organisation and Submodalities}

The experiences we encounter are transformed into trauma because of the organisation of the memory of the said event. We perceive life and store it in our memory through and based on our five senses called modalities, namely, visual, auditory, kinaesthetic, olfactory and gustatory (VAKOG). According to the representation system theory (Bandler \& Grinder, 1979), while the information is stored in the brain and becomes a part of the map in multiple senses and can be retrieved in multiple senses, each of us have a preferred sense(s). So, the initial access to the memories are generally by the primary modality. Hence, the traumatic memory also entails and is led by one or more specific senses mostly visual, auditory and kinaesthetic.

\section{Impact of Trauma}

Trauma has shown to leave a biological impact on people, which includes deregulation and dysfunctioning of the limbic system. The frequency, time duration and many other factors related to a traumatic event, also define the strength of the impact on one's biological functioning. Mental disorders such as depression, anxiety, PTSD and dissociation from reality are comorbid to trauma and may need professional assistance to function in these cases. With varying cortisol levels, the activity of the hypothalamic-pituitary-adrenal (HPA) axis fluctuates; in the cases of chronic and prolonged stress, the baseline HPA response changes and the mechanism alters also depending on the characteristics of the stressors. Moreover, in such cases, the stress responses become more sensitive to low stress types of situations as well (Herman et al., 2016).

Trauma, including one-time, multiple, or longlasting repetitive events, affects everyone differently. Some individuals may clearly display criteria associated with posttraumatic stress disorder (PTSD), but many more individuals will exhibit resilient responses or brief subclinical symptoms or consequences that fall outside of diagnostic criteria. The impact of trauma can be subtle, insidious, or outright destructive. How an event affects an individual depends on many factors, including characteristics of the individual, the type and characteristics of the event(s), developmental processes, the meaning of the trauma, and sociocultural factors (Substance Abuse and Mental Health Services Administration (US), 2014, Ch. 3).

After trauma, it is observed that dysregulation, somatization, fragile self-esteem, and internalised aggression are a common occurrence. When the trauma is rooted to the earlier developmental stages, it perpetuates more gradually and plays an essential role in the personality and its defences, and can trigger unconscious display of actions and situations that have not yet been able to be put into words (Sklarew \& Blum, 2017). Although, PTSD acts as a diagnostic criteria for severe trauma but tends to limit the conversation and invalidate prolonged, repeated and more extreme forms of abuse as they have been noted to carry much complex symptom patterns (such as, alterations in affect regulation, transient dissociative episodes, alterations in self-perception, somatic symptoms, alterations in relations with others, and alterations in systems of meaning are common) than the ones described in the clinical diagnostic criteria of PTSD (Ehrenreich, 2003). It becomes essential to understand the intricacies of the overlapped symptoms and impact of a traumatic incident and further adapt suitable treatment techniques. 
Anchors and Triggers

The daily life of a person with active trauma consists of an intrusive and dysfunctional pattern due to the organisation of the event memory and daily life triggers which make those memories easily accessible. Anchors are what activate our past modalities or sensory data of a particular memory when faced with stimulus to the sensory system at the present moment, therefore taking that past learning and acting in the present. Anchoring is inspired by Pavlov's Classical Conditioning (1902) theory of learning. Classical Conditioning is learning through association developed by Ivan Pavlov (1902) where a combination of two stimuli produce a new learned response in a person or animal. He experimented this theory on his dog, by ringing a bell each time before giving him food. The dog used to salivate once he saw his food but by associating the ringing of the bell with the food, the dog, by the end of the experiment, salivated at the sound of the bell even if no food was given.

The dog was conditioned to think that the bell ringing would result in a bowl of meat. The association is considered reflexive and not a matter of choice. Similarly in trauma, the internalised kinaesthetic or the feeling component gets triggered when faced with elements resembling the organisation of a traumatic memory. The anchors are the environmental and behavioural cues which associate to the past memories and condition us to feel and experience the same memory all over again. Anchors that trigger keep us attached to the belief system we have created around a certain memory or experience and thus we establish a pattern of behaviour when faced with similar stimuli.

\section{Visual/Auditory Kinaesthetic Dissociation: Integrational Distortion and Dissociation}

It is a cardinal need to soften the trauma for an efficient living and better well-being of a person as well as the society. Trauma memories - like all memories - are malleable and prone to distortion. Distortion, (Bandler \& Grinder, 1975) focuses on changing the details of the memory and reorganising the memories formed by the brain in order to change the impact of the events. The submodalities of the memory define the internal representations and the feelings associated with those memories; research shows that our brain often takes references of the representations to understand the feeling associated with it and vice versa. Therefore, if these submodalities are distorted, our brain will also interpret the incident or traumatic event(s) in a different way. Distortion is often done adjacently to dissociation in trauma therapy to create a process involving the disconnection of Visual/Auditory and the Kinaesthetic element of the trauma experiences. Dissociation simply means to disconnect or separate. It is to look at an event from a distance. While dissociating, a separation is achieved between the present and the emotions of trauma. The dissociated perspective not only provides a different point of view, but also creates an emotional distance from the event or the stimulus.

The process called the Visual/Auditory Kinaesthetic Dissociation (V/A-KD) is an intervention including minimal recall of the trauma response along with creations of multiple new associations that, in its nature, rewrite the memory. The traumatic event is either rendered unavailable, severely transformed, or made accessible through a nontraumatic declarative process.(Alberini, 2005; Hupbach, Hardt, Gomez, \& Nadel, 2008; Labar, 2007; Tronel, Milekic, \& Alberini, 2005). It has been studied mainly with reference to the visual and kinaesthetic submodality only but this paper further explores the application of it in the auditory submodality as well due its predominance in masses. Initially, it became a topic of research after appearing in Bandler's Use Your Brain for a Change (1985), which was further expanded in Andreas' Heart of the Mind (Andreas \& Andreas, 1989). The dissociation process consists of the client watching themselves, watching themselves, watching the trauma event as if it were a movie. The image is then further distorted to reorganise the representation. The Visual Kinesthetic Dissociation technique (V/KD), is supported by anecdotal reports by practitioners that cover nearly a quarter century. Among the anecdotal reports are those provided by Richard Bandler, Steve and Connirae Andreas, Robert Dilts, and William McDowell who severally relate that each of them has treated thousands of persons suffering from PTSD and phobic conditions with immediate, lasting results from this short term intervention (Gray, 2011; Andreas \& Andreas, 1989; Bandler, 1985; Dilts \& Delozier, 2000; McDowell \& McDowell, nd). Reconsolidation of Traumatic Memories 
(RTM) is a brief, systematic, non-traumatizing, TFCBT derived from neuro-linguistic programming (NLP) techniques. It is closely related to the Visual Kinesthetic Dissociation protocol (Gray \& Liotta, 2012) and the Rewind Technique. RTM focuses upon the PTSD symptoms that are expressed as immediate, phobic-like responses to triggering stimuli (flashbacks) and repeated nightmares, or night terrors that regularly disrupt sleep and often make it impossible to return to sleep in a normal manner (Gray, Budden-Potts \& Bourke, 2017)

\section{Guilt, Shame and Acceptance}

In a collectivistic society such as India, trauma is followed by the concurrent symptom of guilt and shame in the victim. The emptiness parallel to the trauma, because of the loss of one's security, self, object or some person is magnified by the shame which is used by society to keep people within the cultural limits. It is the disapproval of society, when we don't adhere to societal expectations and norms. We feel it internally and deeply and it is felt publicly. In the cases where the trauma event involves a rather unspoken theme like sex and domestic dysfunction, the systematic and deeprooted shame results in victim blaming because of the society's discomfort of addressing such political themes. Further, this transcends into guilt which acts on a personal level because the victim feels the failure of their own expectations which they form according to societal values. As a result, it is imperative to resolve these aspects of trauma with forgiveness. The long process of forgiving oneself helps in the healing, especially when these emotions are faced in the meta or the dissociated state.

Acceptance and allowance of healing and treatment procedure aids in softening the trauma and helps in the process of dissociation and distortion of the core memories. This paper further explores the possibility of treatment through the above described intervention methods.

\section{Literature Review}

Bessel Van der Kolk, in his book The Body Keeps Score (2014), has noted several points which elaborate on how trauma does not affect a person only on an emotional level. It makes a person unable to trust, have hypervigilance and cannot live a life where their body feels safe literally. He urges to shift from the disease model to a more integrative solution for trauma which focuses on helping them restore relationships, have control over their own bodily responses, be it as simple as that of breathing and be able to communicate suffering. Further, he points out that a lack of control on one's own alert responses as well as any physiological responses may even retraumatize some people (Kolk, 2014). Hence, making it an essential to treat trauma not only emotionally, but also physically and cognitively.

The pioneers of Neurolinguistic Programming have conducted extensive research and published information about the functioning of the brain with respect to one's experiences and how they further complicate to create an elaborate map in the brain (Grinder, Bandler, Andreas \& Andreas, 1979, 1981, 1985, 2013).

Dr. Richard Bandler in his book Use your Mind for a Change (1985), establishes ground for further research into submodalities and addresses the concept of perspectives. He also describes dissociation as a tool used for intense unpleasant memories and phobia. $\mathrm{He}$ introduces the association-dissociation phenomenon classifying them as helpful in cases of trauma.

Connirae Andreas and Steve Andreas (Heart of the Mind, 1989), have written a self-help book by giving an insight to the use of Neurolinguistic Programming and its concepts in different life situations including understanding and healing traumas.

Richard Gray published a review (2011) of two main techniques for the treatment of PTSD and trauma called reconsolidation and extinction and further elaborates on the successful functioning of Visual-Kinesthetic Dissociation Protocol, also known as the Rewind Technique. He has previously conducted pilot studies of three sessions of RTM among male Veterans having high symptom scores on the PTSD Symptom Scale Interview (PSS-I) and the PTSD Checklist - military version (PCL-M) with current-month flashbacks and nightmares (Tylee, Gray, Glatt \& Bourke, 2017) which show that reconsolidation techniques eliminate PTSD diagnoses in upwards of $80 \%$ of those treated. Further, he conducted a randomised controlled trial of 74 male veterans to measure the efficacy of Reconsolidation of Traumatic 
Memories (RTM) which suggested being in line with other studies (Gray et al., 2016; Gray \& Bourke, 2015; Tylee et al., 2017) that the RTM protocol is a viable treatment modality for PTSD-related symptoms in a military population challenged by high levels of intrusive symptoms (Gray, Budden-Potts \& Bourke, 2017).

Treatment Improvement Protocol (TIP) Series, No. 57, Centre for Substance Abuse Treatment (US), Rockville (MD): Substance Abuse and Mental Health Services Administration (US) (2014) have explored and discussed trauma and traumatic stress reactions in the literature review for Trauma-Informed Care in Behavioural Health Services (2014). In the same series they discuss the different dimensions of life that are affected by trauma in the chapter Understanding the Impact of Trauma.

Koziey, P. W., \& McLeod, G. L. (1987) conducted a study with 2 rape victims to treat rape-induced anxiety and related phobia through the Visual-Kinaesthetic Disscoiation procedure and on a preliminary stage suggest using the this technique for treatment of such trauma.

Effectiveness of solution-focused therapy and visual-kinesthetic dissociation on self-concept and adjustment of physically abused adolescents (F. Trinidad, 2000) was investigated with institutionalised 13-17 yearold female physically abused adolescents. The result of this study showed that SFT and VKD are effective in improving the clients' selfconcept and adjustment as recorded at threeday, one-week, and one-month posttests and clinically significant therapeutic effects at onemonth posttest as shown by the elimination of presenting concern and improved adaptive functioning (F. Trinidad, 2000)

These studies carry empirical proof and tested results for the effectiveness of dissociation and distortion procedures in different aspects involving trauma clients and issues that arise parallel to the trauma causing dysfunction in a person's life.

\section{Objective}

The objective of the present research was intended to focus on the following field of study:
To explore the effectiveness of procedures involving integrational dissociation and distortion in treatment of trauma of population with sustained impact of a traumatic event.

a) By studying the effectiveness of techniques with integrational dissociation and distortion in treatment of trauma, we aim to soften the impact of trauma on the population.

b) To acquire an input from a sample of people currently facing the traumatic impact of a certain event in their life about the use of such procedures.

\section{Methodology}

This research paper explores and investigates the efficiency of the V/AKD technique consisting of dissociation and distortion with and of trauma memories. This study design uses a quantitative scale IES-R to attain a reliable and suitable target population. A sample of 234 people was used which further came down to 50. The following sections describe the design, sample selection and procedure in detail.

\section{Sampling Design}

The sample for this research was selected by snowball sampling method and convenience sampling. Firstly, a total number of 234 people, between the age of 18-50 years were tested with the use of Impact of Events Scale (R) to further give a reliable sample of 50 people which fit the required criteria of having present traumatic impact in each of their lives with respect to certain incidents at any point/period of their life. The filtered and chief sample of 50 people was selected based on the scores achieved in the IES(R); everyone who scored above 60 were selected and sent an email for participation, out of which, 50 people responded affirmative for further interview procedure.

\section{Research Instruments}

The scale used to achieve a reliable sample population for the qualitative interview to conduct a demonstration of intervention procedures involving dissociation and distortion was the Impact of Events Scale (R) (IES-R).

\section{Impact of Events Scale (Revised)}

The original version of IES-R is a self-report, short, and easily administered questionnaire to assess PTSD based on the criteria of the 
Diagnostic and Statistical Manual of Mental Disorders, Fourth Edition (DSM-IV) and can be used with both healthy and frail individuals who are exposed to any specific traumatic event (Hamid et al., 2021). It is a 22-item scale consisting of 3 subscales namely, 'hyperarousal', 'avoidance', and 'intrusion' having eight, eight, and six items respectively. The scoring is done on a 5-point Likert Scale from 0 (not at all) to 4 (extremely), which also indicates that the score ranges from $0-88$. The psychometric properties in previously conducted studies (Coffey, Baschnagel et al., 2008; Hamid et al., 2021) show an excellent satisfactory reliability as the Cronbach's alpha coefficients for the three subscales of the IES$\mathrm{R}$ range from 0.84 to 0.95 . The test shows convergent validity with consistent and high correlations between the IES-R total and subscale scores (Coffey, Baschnagel et al., 2008). The convergent validity has also been established in a study of the general Iranian population (Hamid et al., 2021) where the Average Variance Extracted (AVE) is $>0.5$ in all the three subscales.

The research was further conducted with an unstructured interview after the selection and sorting of top 50 scorers on the IES-R showing current prevalence of trauma in their lives.

\section{Visual-Auditory Kinaesthetic Dissociation Technique}

V/AKD (Visual-Auditory Kinaesthetic Dissociation) technique was used for the intervention procedure conducted. In V/AKD, re-experiencing is facilitated by establishing a comfort anchor, in order to allow new learnings while the client is in a dissociated state. It involves viewing the traumatic events from different perspectives to create a less-stressful environment for the client and facilitate the healing process.

The process, consistent with the standard 'Rewind Technique' or the 'movie theatre' technique, started with rapport formation making the client feel safe. After that, they are asked to evoke the traumatic memory until there are visible, but not extreme, physical discomfort cues (heavy breathing, sweating, panic and fear reactions, skin colour and tone etc.). Once noted, they are asked to break-state and come back to the present.
The actual dissociation starts by creating an imaginary visual of the participant sitting in a movie theatre where there is a still image of a safe setting before the event occurred. As this level of dissociation is established, the client is asked to imagine themselves floating away from being seated to behind the plexiglass at the back of the theatre, this creates doubledissociation in the sense that the client is watching themselves, watching themselves on the theatre screen; the traumatic event will watched from this vantage point. They are then asked to slowly start and watch the traumatic event and further change or distort the details of that memory by changing the colour or audio that is involved in the same. They are asked to visualise it till the end until they have a safe image of after the event. This process continues till they can watch it comfortably and without much kinaesthetic discomfort.

The client is then instructed to visualise the end safe image as a starting point and visualise the entire sequence of events in a fast pace and reverse form while being fully associated to the memory.

The effectiveness of the technique is then checked with probing questions and countering all the possible triggers for that event as suggested by Foa and Kozak (Foa \& Kozak, 1986). 


\section{Procedure}

There are two main steps that this research was conducted through. A scale was selected to understand the impact of a negative traumatic event on the participant in the past seven days from taking the test. This study called for the population who are currently going through a significant impact of such an experience where the time lapsed after the event did not hold much importance. The selected scale, IES-R which was developed in 1997 by Daniel Weiss and Charles Marmar, was administered on 234 people out of which 60 were contacted for further participation in the research and finally 50 responded to do so. The IES-R helped in finding the right group of people with significant impact of events to get reliable results for the study.

An unstructured interview with a demo of intervention technique was conducted with training and supervision to collect the suitable data needed to study the effectivity. The data was further analysed and used to derive conclusions for the objective at hand.

\section{Data Processing Tools}

In this qualitative research, the data collected was organised through Inputting, Sorting, Summarization, and Aggregation, and put into a coherent format. The data thus collated was then retrieved, verified, analysed, and interpreted for extraction of relevant information therefrom. The information then was used to derive results and conclusion further.

\section{Data Analysis}

Data analysis was conducted by coding, sorting and noting the recurring themes and responses by the participants in order to verify the effectivity of V/AKD (Visual-AuditoryKinaesthetic Dissociation) Technique involving the distortion of submodalities in a dissociated state. In this research, the responses obtained through the interviews were transcribed. The responses and feedback during the interview and technique demonstration, were followed by a rerun of the same process to validate and cross-examine the answers provided in the first time.

The dominant themes were identified, coded, analysed, and then systematically presented in the given responses for each of the three questions asked in relation to the objective of the study. Coding is a way of indexing or categorising the text in order to establish a framework of thematic ideas about it (Gibbs, 2007). It is the process which involves identifying important words and parts in passages which are relevant to the research objective. The coding categories were created before the process was done; categories like Visual or Auditory Submodalities, emotional release and more were identified.

A parallel was drawn with the participants' responses and the theoretical framework, as well as the literature reviewed for this research which included the work of pre-published research articles, books and pilot studies on the same themes. Lastly, a conclusion was drawn regarding the analysis of the data which helped achieve the objective of understanding the effectiveness of procedures involving integrational dissociation and distortion in treatment of trauma of population with sustained impact of a traumatic event. 


\section{Results}

The research shows a strong efficiency of the V/AKD (Visual-Auditory Kinaesthetic Dissociation) technique. The data shows success of the procedure on 40 out of 50 people on which it was administered.

Table 1 shows the descriptive statistics and results of the IES-R and Figure 1 shows the no. of participants with the dominance of each modality on their memory organisation.

Table 1: Descriptive Statistics of IES-R where $N=216$.

\begin{tabular}{|c|c|c|c|c|c|c|}
\hline & $\begin{array}{l}\text { Minimum } \\
(\mathrm{N}=216)\end{array}$ & $\begin{array}{l}\text { Maximum } \\
(\mathrm{N}=216)\end{array}$ & $\begin{array}{l}\text { Mean } \\
(\mathrm{N}=216)\end{array}$ & $\begin{array}{l}\text { Median } \\
(\mathrm{N}=216)\end{array}$ & $\begin{array}{l}\text { Mode } \\
(\mathrm{N}=216)\end{array}$ & $\begin{array}{l}\text { Std. Deviation } \\
(\sigma)(\mathrm{N}=216)\end{array}$ \\
\hline Total Score & $\mathbf{0}$ & 12 & 6.73 & 7.19 & 5.57 & 2.60 \\
\hline $\begin{array}{l}\text { Avoidance } \\
\text { Subscale }\end{array}$ & $\mathbf{0}$ & 4 & 2.34 & 2.5 & 2.87 & 0.97 \\
\hline $\begin{array}{l}\text { Intrusion } \\
\text { Subscale }\end{array}$ & $\mathbf{0}$ & 4 & 2.10 & 2.28 & 2.71 & 0.92 \\
\hline $\begin{array}{l}\text { Hyperarousal } \\
\text { Subscale }\end{array}$ & $\mathbf{0}$ & 4 & 2.15 & 2.28 & 2.85 & 0.99 \\
\hline
\end{tabular}

Figure 1: Number of interviewed participants and their dominant modalities.

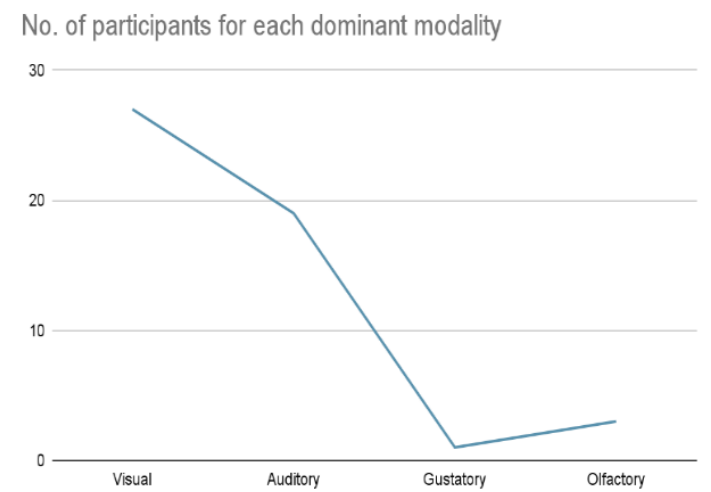

IES-R, a scale with the highest score of 12 , showed an average score of 6.73 in a population of 216, where the average of Avoidance subscale was 2.34, Intrusion subscale was 2.10 and similarly, Hyperarousal subscale was 2.15 . 66 people had a mean score above 8 and thus were selected for further interview process.

Female populations were more prevalent in the test as there were 43 females and 23 males who had a mean score above 8 . The age of the total population ranges from 18 years to 51 years of age; participants under the age of 30 have higher scores than those above 30 years of age. In the selected population of 66, there were 62 people under the age of 30 and 4 people above that.

Similar to studies conducted before (Bandler \& Grinder, 1979), the results show that more than the other modalities (Gustatory, Olfactory), the visual and auditory senses have a higher relevance in the organisation of memories especially in the case of negative memories. Further, 46 out of 50 participants with whom the intervention was conducted, said that their memories have dominantly been saved under the Visual and Auditory modalities.

Additionally, it is to be noted that contrary to previously conducted studies (Bandler \& Grinder, 1979), 4 participants mentioned the predominance of gustatory and olfactory modalities in their memories of a particular traumatic memory. All the participants agreed and reported the strength of the Kinaesthetic (emotional/feeling) component in their memories which got triggered by the environmental stimulus.

Further, the participants below the age of 25 were less reluctant and were open-minded about the intervention procedure compared to the participants above the age of 25 in which the females reported a more profound effect of the intervention conducted than the males 


\section{Discussion}

The data collected in this study was aimed to investigate the efficacy of integrational dissociation and distortion procedures to soften the impact of trauma.

The responses were recorded through an unstructured questionnaire involving a demonstration of the intervention method described in this study. A quantitative research instrument, the Impact of Events Scale (R) was used to filter the participants for this qualitative study and the interview procedure involved three questions with respect to the organisation of the traumatic memory in the brain, followed by the intervention demonstration and finally the first hand account of their experience with the process which provided the adequate results.

The first question was aimed to make the participants aware of the way they experience their memories and which predominant senses have registered the particular traumatic memory in their brain. While the literature and theoretical works have registered and emphasised on the dominance of visual and auditory modalities in organisation of traumatic memories, it is to be noted that 4 out of 50 participants reported other modalities (Olfactory and Gustatory) as an important part of the representation. Further, these were seen coinciding with the visual or auditory (V/A) sense and were never reported as the only way they remembered the memory. Each of the participants were able to identify and most of them noted that they could 'see' or 'hear' the experiences, with Visual having a higher number between the V/A components.

The findings of the second question, which was aimed to explore the feeling or the emotional component of the traumatic memory, showed that people have strong emotional connections with their past memories and these emotions are what make them difficult to deal with. This kinaesthetic component was identified in different manners by the participants where they described the action-reaction occurred at the time the trauma was triggered, both at a physical and an emotional level. A high number reported the immense emotional impact these triggered memories leave them with which are further fueled by, in some cases, anxiety induced physical reactions. The engaging themes here were 'anxiety' 'numbness' 'triggers'.

A demonstration of the V/AKD technique involving dissociation of Kinaesthetic components and distortion of the Visual/Auditory was conducted with the participants after placing suitable disclaimers and creating a safe environment for them. It was followed by an account of their experience with this technique. The second question was repeated in order to check the degree of Kinaesthetic impact the trauma memory had after the procedure. $80 \%$ of the population encountered a noticeable change in their perspective to the negative memory. The responses were also given in a state of surprise as the technique took a quick 10 minutes in some cases with observable changes in the feeling state of participants.

The participants described that this small activity helped them lower the effect that the memories had on them. The changes in the submodalities helped them look at those events in a different light which reduced the emotional involvement in the memory. As shown and stated in the previous literature (Koziey, \& McLeod, 1987; F. Trinidad, 2000) participants have reported a reduction in the overwhelming emotion that were triggered when they thought of a particular or sustained traumatic incident(s). Importantly, Muss has stated (Muss, 2002) that the treatment is appropriate for clients whose major symptoms are severe, abruptly developing experiences of trauma symptoms typically perceived as flashbacks or panic attacks, as Andreas points out (Andreas, 2008, Personal communication).

Hossack and Bental (Hossack \& Bentall, 1996), in their study of five subjects who were treated with a combination of guided visualisations, Jacobsen's deep muscle relaxation and two sessions of the VK/D protocol, state that four out of five which completed the technique reported significant reduction of intrusive images and were able to return to normal life activities.

Although the emotive elements of formerly negative memories have shown to disappear in human investigations of the reconsolidation phenomenon such as this, the events remain accessible on a declarative level, allowing them to be discussed without retraumatizing the 
client. Clients treated with the VK/D protocol retain declarative and episodic access to the stimulus event, but without the traumatic effect (Andreas \& Andreas, 1989; Bandler, 1985; Dilts \& Delozier, 2000; Kindt et al., 2009; Lee, 2009; Lee, Milton, \& Everitt, 2006; Riccio et al., 2006).

\section{Limitations of the Study}

The study is confined and provides evidence based on one session and retest of the integrational distortion and dissociation procedure and does not provide the scope to understand the long-lasting effects of the same, thus having no substance around the extinction.

This study limits the researcher to re-evaluate and understand the long-standing effects of the technique V/AKD and thus does not prove the long-term effectiveness of the same.

Additionally, due to its self-report nature, there is a chance of wrong and altered reporting of the impact of the procedure. Therefore, the window speculation stays open.

The sample size and the convenience and snowballing selection method provides a small number of variation in the population.

\section{Conclusion}

The data collected and analysed in this research reports a high level of effectiveness of the integrational procedure of dissociation and distortion in the treatment of trauma. With a $80 \%$ success rate of this technique, it is suggested to be used further in research and intervention planning with patients of trauma. This evidence helps in improving the facilities provided for curing trauma and shows a concrete plan of action that could be used in the same matter of concern.

Further experimental research is suggested with these techniques on particular sections of the population with specific trauma categories. It is important to further assess the long-lasting effects and efficiency of V/AKD technique across a varied intensity of trauma as well as population. 


\section{References}

Andreas, C., \& Andreas, S. (1989). Heart of the mind. Moab, Utah: Real People Press.

Bacon \& Charles (1981) "Effects of visual-kinesthetic dissociation on anxiety in six phobic clients". Graduate Student Theses, Dissertations, \& Professional Papers. 4954. https://scholarworks.umt.edu/etd/4954

Bandler, R., Andreas, S., \& Andreas, C. (1998). Using your brain--for a change. Moab, Utah: Real People Press. Bruce H. Sklarew \& Harold P. Blum (2006) Trauma and depression, The International Journal of Psychoanalysis, 87:3, 859-861, DOI: 10.1516/14P5-CYAN-MPL8-6RL4

Centre for Substance Abuse Treatment (US). Trauma-Informed Care in Behavioural

Health Services. Rockville (MD): Substance Abuse and Mental Health Services Administration (US); 2014. (Treatment Improvement Protocol (TIP) Series, No. 57.) Chapter 3 , Understanding the Impact of Trauma. Available from: https://www.ncbi.nlm.nih.gov/books/NBK207191/

Center for Substance Abuse Treatment (US). Trauma-Informed Care in Behavioral Health Services. Rockville (MD): Substance Abuse and Mental Health Services Administration (US); 2014. (Treatment Improvement Protocol (TIP) Series, No. 57.) Section 1, A Review of the Literature. Available from: https://www.ncbi.nlm.nih.gov/books/NBK207192/

Dilts, R., \& Delozier, J. (2000). The Encyclopedia of Systemic Neuro-Linguistic

Programming and NLP New Coding. Retrieved January 2, 2022, from

www.nlpu.com.

Ehrenreich, J. (2003). Understanding PTSD: Forgetting

"Trauma". Analyses Of Social Issues And Public Policy, 3(1), 15-28. doi: 10.1111/j.1530-2415.2003.00012.x

Foa, E. B., \& Kozak, Michael J. (1986). Emotional Processing of Fear: Exposure to

Corrective Information. Psychological Bulletin, Vol. 99, No. 1.

Gray, R., \& Liotta, R. (2012). PTSD: Extinction,

reconsolidation, and the visual-kinesthetic dissociation protocol. Traumatology, 18(2), 3-16. doi:

$10.1177 / 1534765611431835$

Gray, R., Budden-Potts, D., \& Bourke, F. (2017).

Reconsolidation of Traumatic Memories for PTSD: A

randomised controlled trial of 74 male veterans. Psychotherapy

Research, 29(5), 621-639. doi:

$10.1080 / 10503307.2017 .1408973$

Herman, J. P., McKlveen, J. M., Ghosal, S., Kopp, B., Wulsin, A., Makinson, R., Scheimann, J., \& Myers, B. (2016). Regulation of the Hypothalamic-Pituitary-Adrenocortical Stress Response. Comprehensive Physiology, 6(2), 603-621. https://doi.org/10.1002/cphy.c150015

Hossack, A., \& Bentall, R. P. (1996). Elimination of posttraumatic symptomatology by

relaxation and visual-kinesthetic dissociation. Journal of Traumatic Stress, 9(1), 99-110.

Kindt, M., Soeter, M., \& Vervliet, B. (2009). Beyond extinction: erasing human fear responses and preventing the return of fear Nat Neurosci, 12(3), 256-258..
Kolk, B., \& Fisler, R. (1995). Dissociation and the fragmentary nature of traumatic memories: Overview and exploratory study. Journal Of Traumatic Stress, 8(4), 505-525. doi: $10.1007 / \mathrm{bf} 02102887$

Kolk, B., 2014. The Body Keeps the Score. Viking Press.

Koziey, P., \& McLeod, G. (1987). Visual-kinesthetic dissociation in treatment of victims of rape. Professional Psychology: Research And Practice, 18(3), 276-282. doi: 10.1037/0735-7028.18.3.276

Lee, J. L. C. (2009). Reconsolidation: maintaining memory relevance. Trends in Neurosciences, 32(8), 413-420

Lee, J. L. C., Milton, A. L., \& Everitt, B. J. (2006). Reconsolidation and Extinction of Conditioned Fear: Inhibition and Potentiation. J. Neurosci., 26(39), 10051-10056.

Muss, D. (2002). The Rewind Technique In the treatment of Post-Traumatic Stress

Disorder: Methods and Application In C. R. Figley (Ed.), Brief Treatments for the

Traumatised (pp. 306-314). West Port, Conn: Greenwood Press.

Passmore, J. and Rowson, T. S. (2019) Neuro-linguistic programming: a critical review of $N L P$ research and the application of NLP in coaching. International Coaching Psychology Review, 14 (1). pp. 57-69. ISSN 2396-8753 Available at https://centaur.reading.ac.uk/91275/

Rash, C., Coffey, S., Baschnagel, J., Drobes, D., \& Saladin, M. (2008). Psychometric properties of the IES-R in traumatised substance dependent individuals with and without PTSD. Addictive Behaviours, 33(8), 1039-1047. doi: 10.1016/j.addbeh.2008.04.006

Riccio, D. C., Millin, P. M., \& Bogart, A. R. (2006). Reconsolidation: A brief history, a retrieval view, and some recent issues. Learning \& Memory, 13(5), 536-544.

Sharif Nia, H., Kaur, H., Fomani, F., Rahmatpour, P., Kaveh, O., \& Pahlevan Sharif, S. et al. (2021). Psychometric Properties of the Impact of Events Scale-Revised (IES-R) Among General Iranian Population During the COVID-19 Pandemic. Frontiers In Psychiatry, 12. doi: 10.3389/fpsyt.2021.692498

Trinidad, M. (2000). Effectiveness of solution-focused therapy and visual-kinesthetic dissociation on self-concept and adjustment of physically abused adolescents. Retrieved 28 December 2021, from https://animorepository.dlsu.edu.ph/etd doctoral/859/

Tylee, D., Gray, R., Glatt, S., \& Bourke, F. (2017). Evaluation of the reconsolidation of traumatic memories protocol for the treatment of PTSD: a randomised, wait-list-controlled trial. Journal Of Military, Veteran And Family Health, 3(1), 21-33. doi: $10.3138 /$ jmvfh. 4120

Wallace, J., 2020. [Blog] Trauma is Subjective, Available at: <https://arcmonroe.org/trauma-is-subjective/> [Accessed 1 February 2022]. 